\title{
2.5D far-field diffraction tomography inversion scheme for GPR that takes into account the planar air-soil interface
}

\author{
Meincke, Peter
}

Published in:

Proceedings of IEEE Antennas and Propagation Society International Symposium

Link to article, DOI:

10.1109/APS.2001.959811

Publication date:

2001

Document Version

Publisher's PDF, also known as Version of record

Link back to DTU Orbit

Citation (APA):

Meincke, P. (2001). 2.5D far-field diffraction tomography inversion scheme for GPR that takes into account the planar air-soil interface. In Proceedings of IEEE Antennas and Propagation Society International Symposium (Vol. 2, pp. 662-665). IEEE. https://doi.org/10.1109/APS.2001.959811

\section{General rights}

Copyright and moral rights for the publications made accessible in the public portal are retained by the authors and/or other copyright owners and it is a condition of accessing publications that users recognise and abide by the legal requirements associated with these rights.

- Users may download and print one copy of any publication from the public portal for the purpose of private study or research.

- You may not further distribute the material or use it for any profit-making activity or commercial gain

- You may freely distribute the URL identifying the publication in the public portal 


\title{
2.5-D Far-Field Diffraction Tomography Inversion Scheme for GPR That Takes into Account the Planar Air-Soil Interface
}

Peter Meincke ${ }^{1}$

Ørsted.DTU, Section for Electromagnetic Systems, Build. 348

Technical University of Denmark, DK-2800 Kgs. Lyngby, Denmark

pme@emi.dtu.dk

\begin{abstract}
A new 2.5-D inversion scheme is derived for fixed-offset ground penetrating radar (GPR) that takes into account the planar air-soil interface. The inversion scheme is based upon the first Bom approximation and a far-field ap-
\end{abstract} proximation of the dyadic Green function for a two-layer medium.

1. Introduction

Linear inversion schemes based upon the concept of diffraction tomography have proven successful for ground penetrating radar (GPR) imaging [1,2]. As illustrated in [2] it is important to incorporate in the inversion the presence of the air-soil interface. The inversion scheme in [2] was derived by inserting the plane-wave expansion of the two-layer dyadic Green function into the linearized Lippmann-Schwinger integral equation and performing an asymptotic expansion valid when the object is located deep in the soil and finally using the inverse Fourier transform. This procedure requires that the soil is lossless. It was shown in [2] that the procedure could be heuristically modified to include loss. However, since this heuristic modification is not exact, it gives rise to artifacts in the image.

In this paper another approach is presented to derive an inversion scheme for GPR that takes into account the planar air-soil interface. Here, the far-field approximation of the dyadic Green function for the two-layer medium is used rather than applying the asymptotic procedure outlined in [2]. Since the inverse Fourier transform is still applied to carry out the inversion, it must again be assumed in the derivation that the soil has no loss. Nevertheless, an heuristic modification is possible to include the loss and this modification does not give rise to artifacts in the image as was the case in [2]. A similar result was derived in [3] by using the generalized Radon transform. However, the inverse scheme to be presented below is more efficient than that in [3] since it can be implemented using fast Fourier transforms (FFT's)

For simplicity only the 2.5 -D case is considered, that is, it is assumed that the scattered object in the soil is invariant in one direction, which for instance is the case for a pipe. Throughout the paper the time factor $\exp (-i \omega t)$ is assumed and suppressed.

\section{The 2.5-D Forward Model}

An example of the GPR configuration involving the planar air-soil interface is shown in Figure 1. A Cartesian $x y z$ coordinate system is introduced such that the

'The Danish Technical Research Council is acknowledged for supporting this work. 
$x y$ plane coincides with the interface and such that $z>0$ is air. An object, which is assumed infinitely long in the $\hat{\mathbf{x}}$ direction, is buried in the soil. The propagation constant of air is $k_{0}=\omega \sqrt{\mu_{0} \epsilon_{0}}$ and that of soil is $k_{1}=\sqrt{\omega^{2} \mu_{0} \epsilon_{1}+i \omega \mu_{0} \sigma_{1}}$. The position of the receiving antenna is described by $\mathbf{r}_{r}=\mathbf{R}_{\tau}+\hat{\mathbf{z}} z_{r}$ and that of the transmitting antenna is $\mathbf{r}_{t}=\mathbf{r}_{r}+\mathbf{r}_{\Delta}$ with the fixed offset $\mathbf{r}_{\Delta}=\mathbf{R}_{\Delta}+\hat{\mathbf{z}} z_{\Delta}$. It is assumed that the contrast in conductivity $\Delta \sigma(\mathbf{r})=\sigma(\mathbf{r})-\sigma_{1}$ is much less than the contrast in permittivity, i.e., $\Delta \sigma(\mathbf{r}) \ll \omega \Delta \epsilon(\mathbf{r})$ over the frequency band of interest $\omega_{\min }<\omega<\omega_{\max }$. Then, by assuming ideal dipole antennas, the output $s_{0}$ of an $\dot{x}$-directed receiving antenna due to a transmitting antenna with dipole moment $\hat{\mathbf{x}} I(\omega)$ can be related to $\Delta \epsilon(\mathbf{r})$ as $[2,(11)]$

$$
s_{0}\left(\mathbf{r}_{r}, \omega\right)=i \omega^{3} \mu_{0}^{2} I(\omega) \int_{V} \hat{\mathbf{x}} \cdot \overline{\mathbf{G}}\left(\mathbf{r}_{r}, \mathbf{r}^{\prime}, \omega\right) \cdot\left[\hat{\mathbf{x}} \cdot \overline{\mathbf{G}}\left(\mathbf{r}_{t}, \mathbf{r}^{\prime}, \omega\right)\right] \Delta \epsilon\left(\mathbf{r}^{\prime}\right) d^{3} \mathbf{r}^{\prime}
$$

where the dyadic Green function for the interface can be written as

$$
\overline{\mathbf{G}}\left(\mathbf{r}, \mathbf{r}^{\prime}, \omega\right)=\frac{i}{8 \pi^{2}} \int_{-\infty}^{\infty} \overline{\mathbf{F}}(\mathbf{K}, \omega) \exp \left(i \gamma_{0}(\mathbf{K}) z\right) \exp (i R \Phi(\mathbf{K})) d^{2} \mathbf{K}
$$

where $z>0, z^{\prime}<0, \mathbf{K}=\hat{\mathbf{x}} k_{x}+\hat{\mathbf{y}} k_{y}, \gamma_{i}(\mathbf{K})=\sqrt{k_{i}^{2}-k_{x}^{2}-k_{y}^{2}}, i=0,1$, $\Phi(\mathbf{K})=k_{x} \frac{x-x^{\prime}}{R}+k_{y} \frac{y-y^{\prime}}{R}+\gamma_{1}(\mathbf{K}) \frac{\left|z^{\prime}\right|}{R}$, and $R=\sqrt{\left(x-x^{\prime}\right)^{2}+\left(y-y^{\prime}\right)^{2}+z^{\prime 2}}$. The expression for the dyadic $\overline{\mathbf{F}}(\mathbf{K})$ can be found in $[2,(6)]$. We now focus on the 2.5-D case in which the buried object is invariant in the $\hat{\mathrm{x}}$-direction, i.e. $\Delta \epsilon(\mathbf{r})=\Delta \epsilon(y, z)$. To this end the far-field expression of $\overrightarrow{\mathbf{G}}$, that is, the asymptotic expansion as $R \rightarrow \infty$, is inserted into (1). For $x_{r}=0$ this yields

$$
s_{0}\left(y_{r}, \omega\right)=\frac{i \omega^{3} \mu_{0}^{2} I(\omega)}{(4 \pi)^{2}} \int_{-\infty}^{\infty} \int_{-\infty}^{\infty} F\left(y_{r}, y^{\prime}, z^{\prime}, \omega\right) \Delta \epsilon\left(y^{\prime}, z^{\prime}\right) d y^{\prime} d z^{\prime}
$$

where

$$
\begin{gathered}
F\left(y_{r}, y^{\prime}, z^{\prime}, \omega\right)=\int_{-\infty}^{\infty}\left(\hat{\mathbf{x}} \cdot \tilde{\mathbf{F}}\left(\mathbf{K}_{r}, \omega\right)\right) \cdot\left[\hat{\mathbf{x}} \cdot \overline{\mathbf{F}}\left(\mathbf{K}_{t}, \omega\right)\right] \gamma_{1}\left(\mathbf{K}_{r}\right) \gamma_{1}\left(\mathbf{K}_{t}\right) \\
\cdot \exp \left(i\left[\gamma_{0}\left(\mathbf{K}_{r}\right) z_{r}+\gamma_{0}\left(\mathbf{K}_{t}\right) z_{t}\right]\right) \frac{\exp \left(i k_{1}\left[R_{r}+R_{t}\right]\right)}{R_{r} R_{t}} d x^{\prime}
\end{gathered}
$$

where $R_{r}=\sqrt{x^{\prime 2}+\rho_{r}^{2}}, \rho_{r}=\sqrt{\left(y_{r}-y^{\prime}\right)^{2}+{z^{\prime}}^{2}}, \mathbf{K}_{r}=\hat{\mathbf{x}} k_{x r}+\hat{\mathbf{y}} k_{y r}=\hat{\mathbf{x}} k_{1} \frac{-x^{\prime}}{R_{r}}+$ $\hat{\mathbf{y}} k_{1} \frac{y_{r}-y^{\prime}}{R_{r}}$, and similarly for $R_{t}$ and $\mathbf{K}_{t}$. Using the substitution $u=\rho_{r}^{-1} x^{\prime}, F$ in (4) takes on the form $\int_{-\infty}^{\infty} F_{u}(u) \exp \left(i k_{1} \rho_{r}\left[\sqrt{1+u^{2}}+\sqrt{\rho_{t}^{2} / \rho_{r}^{2}+u^{2}}\right]\right) \rho_{r} d u$. A simple asymptotic calculation as $\rho_{r} \rightarrow \infty$ then gives $F \sim F_{u}(0) \exp \left(i k_{1}\left[\rho_{r}+\right.\right.$ $\left.\left.\rho_{t}\right]\right) \exp \left(i \frac{\pi}{4}\right) \sqrt{2 \pi \rho_{r}} /\left(k_{1}\left(1+\rho_{r} / \rho_{t}\right)\right)$. Following the procedure of [1] $\rho_{r}$ and $\rho_{t}$ are next expanded around a fixed point $o$ in the vicinity of the object and with

663 
position vector $\rho_{o}$ as $\rho_{r} \approx \rho_{r o}-\hat{\rho}_{r o} \cdot\left(\boldsymbol{\rho}^{\prime}-\boldsymbol{\rho}_{o}\right) \approx \rho_{r o}$ with $\rho_{r o}=\rho_{r o} \hat{\boldsymbol{\rho}}_{r o}=$ $\hat{\mathbf{y}}\left(y_{r}-y_{o}\right)-\hat{\mathbf{z}} z_{o}$, and similarly for $\rho_{t}$. This yields

$$
s_{o}\left(y_{r}, \omega\right)=D\left(y_{r}, \omega\right) \exp \left(i k_{1} \boldsymbol{\rho}_{o} \cdot\left[\hat{\boldsymbol{\rho}}_{r o}+\hat{\boldsymbol{\rho}}_{t o}\right]\right) \widetilde{\Delta \epsilon}\left(k_{1}\left[\hat{\boldsymbol{\rho}}_{r o}+\hat{\boldsymbol{\rho}}_{t o}\right]\right)
$$

where $\Delta \tilde{\epsilon}$ is the 2-D spatial Fourier transform of $\Delta \epsilon$ and

$$
\begin{gathered}
D\left(y_{r}, \omega\right)=\frac{i \omega^{3} \mu_{0}^{2} I(\omega) z_{o}^{2} \exp \left(i \frac{\pi}{4}\right)}{2 \pi \sqrt{2 \pi k_{1} \rho_{r o} \rho_{t o}\left(\rho_{r o}+\rho_{t o}\right)}} \exp \left(i k_{1}\left[\rho_{r o}+\rho_{t o}\right]\right) \\
\cdot \exp \left(i k_{1}\left[\sqrt{\left(\frac{k_{0}}{k_{1}}\right)^{2}+\left(\frac{z_{o}}{\rho_{r o}}\right)^{2}-1 z_{r}}+\sqrt{\left(\frac{k_{0}}{k_{1}}\right)^{2}+\left(\frac{z_{o}}{\rho_{t o}}\right)^{2}-1 z_{t}}\right]\right) \\
\left(\sqrt{\rho_{r o}^{2}\left[\left(\frac{k_{0}}{k_{1}}\right)^{2}-1\right]+z_{o}^{2}+\left|z_{o}\right|}\right)^{-1}\left(\sqrt{\rho_{t o}^{2}\left[\left(\frac{k_{0}}{k_{i}}\right)^{2}-1\right]+z_{o}^{2}}+\left|z_{o}\right|\right)_{(6)}^{-1}
\end{gathered}
$$

3. Inversion

Using the fact that $\Delta \epsilon$ is real, this function can be found from its Fourier transform through

$$
\Delta \epsilon\left(\boldsymbol{\rho}_{o}\right)=\frac{1}{2 \pi^{2}} \operatorname{Re}\left[\int_{-\infty}^{\infty} \int_{-\infty}^{\infty} \widetilde{\Delta \epsilon}(\mathbf{K}) \exp \left(i \mathbf{K} \cdot \boldsymbol{\rho}_{o}\right) d^{2} \mathbf{K}\right] .
$$

To use the relation between the measured data $s_{o}$ and $\widetilde{\Delta \epsilon}$ in (5) we must assume that $k_{1}$ is real. Then, substituting in (7) $\mathbf{K}=k_{1}\left(\hat{\boldsymbol{\rho}}_{\text {ro }}+\hat{\boldsymbol{\rho}}_{t o}\right)$, and using that

$d^{2} \mathbf{K}=J\left(y_{r}, \omega\right) d y_{r} d \omega=\left|\frac{k_{1}^{2} z_{o}\left(\rho_{r o}^{2}+\rho_{t o}^{2}\right)}{\omega \rho_{r o}^{2} \rho_{t o}^{2}}\left(1+\frac{z_{o}^{2}+\left(y_{o}-y_{r}\right)\left(y_{o}-y_{t}\right)}{\rho_{r o} \rho_{t o}}\right)\right| d y_{r} d \omega$

we obtain

$$
\Delta \epsilon\left(\boldsymbol{\rho}_{o}\right)=\frac{1}{2 \pi^{2}} \operatorname{Re}\left[\int_{0}^{\omega_{\max }} \int_{y_{\min }}^{y_{\max }} \frac{s_{o}\left(y_{r}, \omega\right) J\left(y_{r}, \omega\right)}{D\left(y_{r}, \omega\right)} d y_{r} d \omega\right] .
$$

Since the dependence of the factor $J\left(y_{r}, \omega\right) / D\left(y_{r}, \omega\right)$ on $y_{r}$ is of the form $y_{o}-y_{r}$, the integration over $y_{r}$ is a convolution and thus, it can be calculated efficiently using FFT's.

\section{Numerical Example}

The inversion scheme of Section 3 is now tested on synthetic GPR data. Figure 1 shows a dielectric pipe with outer diameter $16 \mathrm{~cm}$ and inner diameter $8 \mathrm{~cm}$ located $1 \mathrm{~m}$ below the interface. The conductivities of the soil and the two regions of the pipe are $0.005 \mathrm{~S} / \mathrm{m}$. The permittivities of the soil, the inner and outer regions of the pipe are $8 \epsilon_{0}, 8.1 \epsilon_{0}$, and $8.2 \epsilon_{0}$, respectively. The synthetic GPR data

664 


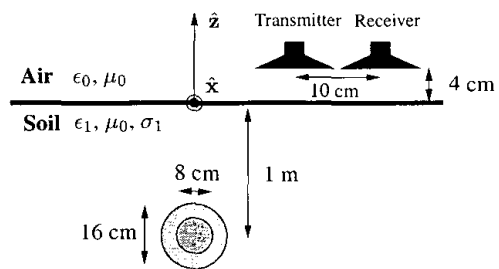

Figure 1: The configuration involving a two-region pipe.

is calculated from an eigenfunction expansion. It is assumed that the radar uses 60 frequencies equally spaced in the range $20 \mathrm{MHz}<f<1.3 \mathrm{GHz}$. Figure 2 shows the image of $\Delta \epsilon(y, z) / \epsilon_{0}$ obtained from (9), heuristically modified to in-

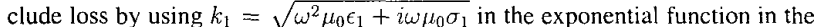
first line of (6). It is noted that there are no severe artifacts in the image below the pipe as was the case in [2].

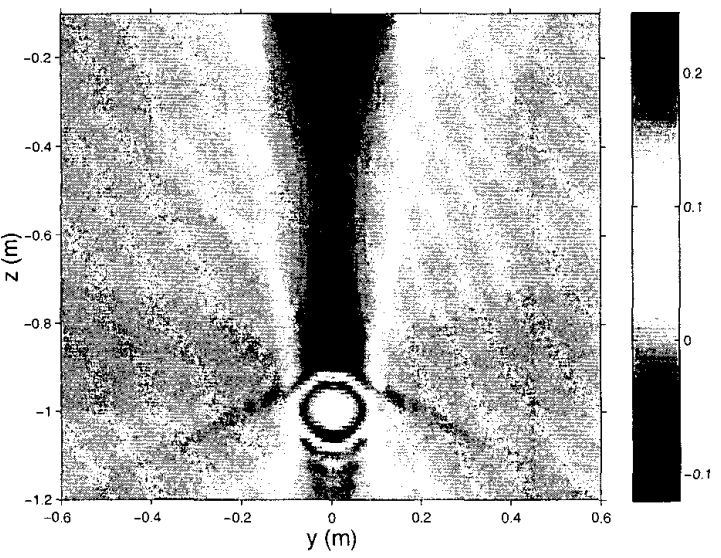

References $\quad$ Figure 2: The image of $\Delta \epsilon(y, z) / \epsilon_{0}$.

[1] J. Molyneux and A. Witten, "Diffraction tomographic imaging in a monostatic measurement geometry," IEEE Trans. Geosci. Remote Sensing, vol. 31 pp. 507-511, Mar. 1993.

[2] T. Hansen and P. Meincke Johansen, "Inversion scheme for ground penetrating radar that takes into account the planar air-soil interface." IEEE Trans. Geosci. Remote Sensing, vol. 38, pp. 496-506, Jan. 2000.

[3] T. Wang and M.L. Oristaglio, "GPR imaging using the generalized Radon transform," Geoplyysics, vol. 65, pp. 1553-1559, Sept.-Oct. 2000. 